\title{
Air, Air Everywhere!
}

\author{
Rafiq Kanji ${ }^{1}$, Kayur Patel ${ }^{1}$, and Douglas Stangoe ${ }^{2}$ \\ ${ }^{1}$ East Surrey Hospital \\ ${ }^{2}$ King's College Hospital NHS Trust
}

July 26, 2020

\begin{abstract}
This chext x-ray (CXR) shows a large right sided pneumothorax with a degree mediastinal shift suggesting tensioning (The CXR you should never see!). Furthermore, features of pneumomediastinum are present including the continuous diaphragm sign, and subcutaneous emphysema around the neck and right side of the thorax
\end{abstract}

\section{Key Clinical Message:}

When using a GEB, a common instrument used to aid anesthetists with difficult intubations, one must appreciate the significant consequences that can arise, be prepared to manage any emergency complications and use the device cautiously and safely

\section{Description:}

A 53-year-old African-Caribbean male patient, with a background of hypertension, tested positive for COVID-19 and was intubated and ventilated on the intensive care unit. He had a classical COVID-19 chest x-ray consisting of hazy consolidation and ground-glass opacification bilaterally. During his admission, he developed an endotracheal tube (ETT) cuff leak. The ETT exchange, done with a gum elastic bougie (GEB), was challenging due to the high volume of thick secretions distorting the view of the glottic inlet. As demonstrated, this difficulty caused a significant right-sided pneumothorax with surgical emphysema secondary to a pneumomediastinum, likely caused by the GEB. Additionally, there is a degree of mediastinal shift, though this is difficult to assess due to patient rotation. What is the immediate management? Ideally, such as in this case, an emergency surgical chest drain should be inserted concomitantly so as not to exacerbate the pneumothorax and cause tensioning as well as further haemodynamic instability (Goswami \& Sarangi, 2016). The primary teaching point is the significant consequences that can be caused by a GEB, and while a useful instrument common to anesthetic practice, it should be used cautiously and safely.

\section{Author Contributions:}

Dr. Kanji was involved in the patient's care during their time on Intensive Care and was able to get the necessary clinical details and images required to submit this clinical image. Additionally, he was involved in writing and assembling the manuscript.

Dr. Patel was involved in writing the case report and ensuring the clinical image was of an appropriate resolution.

Dr. Stangoe was involved in writing the case report.

\section{Conflicts of Interest:}

No author has any conflicts of interest with regards to this case / publication 


\section{References:}

Goswami, U., \& Sarangi, S. (2016). Iatrogenic surgical emphysema and pneumomediastinum in a case of emergency lower segment caesarean section. Indian Journal of Anaesthesia , 60 (2), 6-8.

\section{Figure Legend:}

This chext x-ray shows a large right sided pneumothorax with a degree mediastinal shift suggesting tensioning (The chest x-ray you should never see!). Furthermore, features of a pneumomediastinum are present including the continuous diaphragm sign, air around the aortic arch and bilateral subcutaneous emphysema around the neck and the right side of the thorax

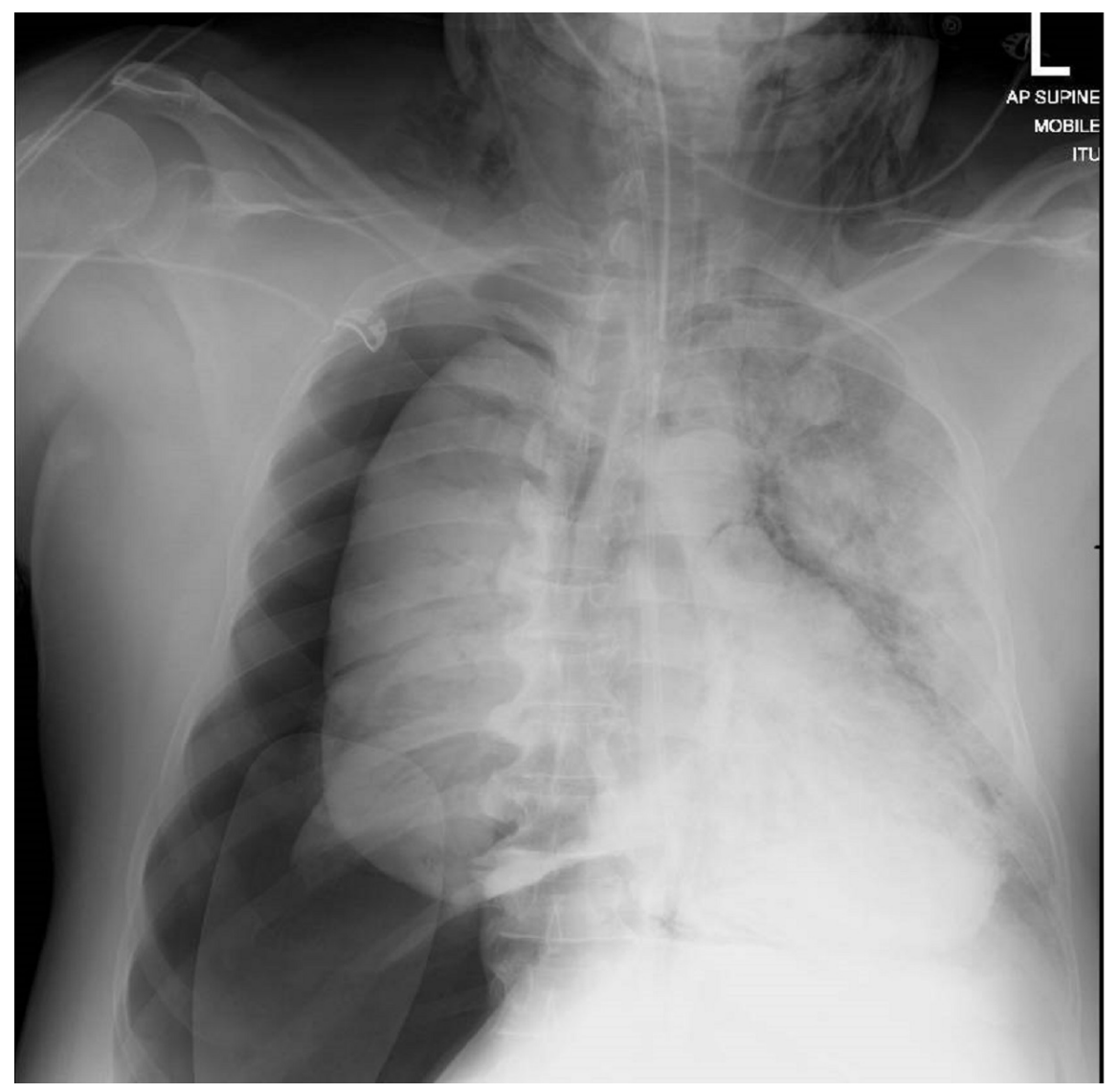

